\title{
The Consequences of Earlier Reproduction in Declining Populations
}

\author{
CHRISTOPHER G. HOOGENDYK* \\ School of Natural Resources, The University of Michigan, Ann Arbor, Michigan 48109 \\ AND \\ GEORGE F. ESTABROOK \\ Division of Biological Sciences, The University of Michigan, Ann Arbor, Michigan 48109 \\ Received 18 May 1984; revised 25 May 1984
}

\begin{abstract}
Conflicting claims have been published concerning the consequences of earlier reproduction. In particular, it has been claimed that earlier reproduction will increase the rate of decline in a declining population, have no effect in a stable population, and enhance the rate of growth in an increasing population. However, if fecundity and mortality are conceptually separated so that earlier reproduction is defined as moving the fecundity column of the life table forward in time but not changing the mortality column, then earlier reproduction will nearly always cause the rate of change of a population to become more positive. This definition is believed to be more consistent with what biologists think of as earlier reproduction. Also, this concept requires that the cost of reproducing earlier be explicitly addressed rather than hiding it in assumptions implicit in a definition of earlier reproduction. This paper reconciles published mathematical demography models with published biological claims concerning earlier reproduction.
\end{abstract}

\section{INTRODUCTION}

There have been several sources of the claim that earlier reproduction causes a declining population to decline more rapidly. It usually arises from the broad interdependence of all the parameters and variables that can be derived from life tables and that describe the behavior of a population. For example, a life table or equation may occur in which the mortality rates and fertility rates are tied together by the form of the variables. Then, when the age of reproduction is changed, the mortality rates are affected as well. The

*Current address: Terrestrial and Aquatic Ecology Division, Brookhaven National Laboratory, Upton, New York 11973; (516) 282-3744.

Elsevier Science Publishing Co., Inc., 1984 
result may be a simultaneous shift of both the mortality rates and the fertility rates. In one case, this had the effect of compressing the time scale by multiplying the exponential rate of increase by some positive factor; with the time frame compressed, an increasing population increases faster and a declining population declines faster.

A similar source of confusion arises from the analogy with interest rates. The term $e^{-r \cdot x}$ occurs in many demographic equations and has been described as applying a discount rate $r$ to offspring at age $x$. This analogy leads to discussions concerning the selective advantage of having offspring early in life or late in life based on $r$, the exponential rate of increase, serving as a discount rate. Such discussions neglect the fact that by having offspring earlier in life or later in life, the life table is changed, and $r$, which is a function of the life table, may also change. The equations on which such discussions are based assume a constant life history and a stable age distribution. When the life history is changed, other parts of the equation also change to maintain the relationships among the variables.

The presentation of the specific examples below will assume some familiarity with the mathematical methods used in studying life histories. A good introduction may be found in Ricklefs [33] and Mertz [26].

\section{EXAMPLE I}

One of the earliest proponents of the idea that earlier reproduction increases the rate of decline in a declining population is Mertz [27-29]. In his paper on the mathematical demography of the California condor, Mertz [27] presents an analysis of the effect of development time. He begins with a life history table for the California condor (Table 1). In this table, $\beta$ is the probability at birth of surviving to the age of first reproduction and $\rho$ is the annual adult survival rate. The birds mature at age 5 and lay one egg every other year. One half of the eggs are female.

From any life history table, the growth rate can be determined using Lotka's [24] classic equation:

$$
\sum_{x=0}^{\infty} \lambda^{-x} l_{x} m_{x}=1,
$$

where $\lambda\left(=e^{r}\right)$ is the geometric rate of increase, and $r$ is the exponential rate of increase, of a population that has a stable age distribution and constant life history parameters as defined by $l_{x}$ and $m_{x}$. Substituting the entries from Table 1 into Equation (1), Mertz arrives at the following:

$$
\lambda^{5}-\rho^{2} \lambda^{3}-\frac{\beta}{2}=0
$$


TABLE 1

The Basic California Condor Life History ${ }^{a}$

\begin{tabular}{cccll}
\hline$x$ & $l_{x}$ & $m_{x}$ & $l_{x} m_{x}$ & \\
\hline 0 & 1.0 & 0 & 0 & \\
1 & $\ldots$ & 0 & 0 & \\
2 & $\ldots$ & 0 & 0 & \\
3 & $\cdots$ & 0 & 0 & \\
4 & $\cdots$ & 0 & 0 & \\
5 & $\beta$ & $\frac{1}{2}$ & $\beta / 2$ & \\
6 & $\beta \rho$ & 0 & 0 & \\
7 & $\beta \rho^{2}$ & $\frac{1}{2}$ & $\beta \rho^{2} / 2$ & (for $i$ odd) \\
$i$ & $\beta \rho^{i-5}$ & $\frac{1}{2}$ & $\beta \rho^{i-5} / 2$ & (for $i$ even) \\
\hline
\end{tabular}

a $x=$ age in years, $l_{x}=$ probability at birth of living to age $x$, and $m_{x}=$ the expected number of daughter eggs laid at age $x$ by a surviving female. From Mertz [27].

This equation is used to construct figures with isopleths of $\lambda$ as a function of $\beta$ and $\rho$.

Examining the effects of development time, Mertz [27, p. 445] writes, "For fixed values of $\beta$ and $\rho$, development time has no effect on the equation for $R_{0}\left[R_{0}=\sum_{x=0}^{\infty} l_{x} m_{x}=\beta /\left(2\left(1-\rho^{2}\right)\right)\right]$. However, $\lambda$ is affected, and signifying development time by the symbol $\tau$, a generalization for equation (2) is obtained:

$$
\lambda^{\tau}-\rho^{2} \lambda^{\tau-2}-\frac{\beta}{2}=0
$$

where $\tau$ is an integer 2 or greater." Equation (2c) is used with $\tau=4$ to construct additional isopleths on the figure containing Equation (2) isopleths [see Figure 1(a)]. If the dashed isopleth $(\tau=4)$ is below the solid isopleth $(\tau=5)$, this is interpreted as meaning that when $\tau=4$ a lower juvenile and/or adult survival rate can result in the samc ratc of changc for the population, or, if the survival rates remain the same, then the rate of change of the population will become more positive. From Figure 1(a) Mertz concludes that "for declining populations... the shorter developmental time accelerates the population decline." Mertz then argues that rapid development would be selected against during population declines. In applying this and other conclusions to the California condor, he cites evidence for population decline through a long span of geologic time and argues [28] that "the California condor may have had its life history shaped by many years of population contraction in the absence of many colonizing opportunities." 
Mertz's Equations (2) and (2c) are special cases for the particular entries in the life table. When he begins to vary $\tau$, he neglects the fact that $\beta$ is a survival rate and that the time portion of $\beta$ is $\tau$. Changing $\tau$ from 5 to 4 not only increases the rate of maturation but also increases the mortality rate of immature birds by the same factor. The $l_{x}$ column and the $m_{x}$ column of the life table are both moved up one year. This shifting of both columns together leaves $R_{0}$ unchanged and the direction of population growth or decline unchanged, but increases the rate at which the population is either growing or declining.

To see the effect of early development without imposing a higher rate of mortality as well, $\beta$ must be broken down into a yearly rate. Mertz [27] says, "a heavy proportion of immature mortality probably falls in the first year of life." Taking the simplest approach, $\beta$ can be replaced with $\beta_{1} \rho^{\top}{ }^{1}$, in which $\beta_{1}$ is first year survival, and survival in successive years is the same as for adults. Goodman [15] used this same approach in examining the life history

(a)

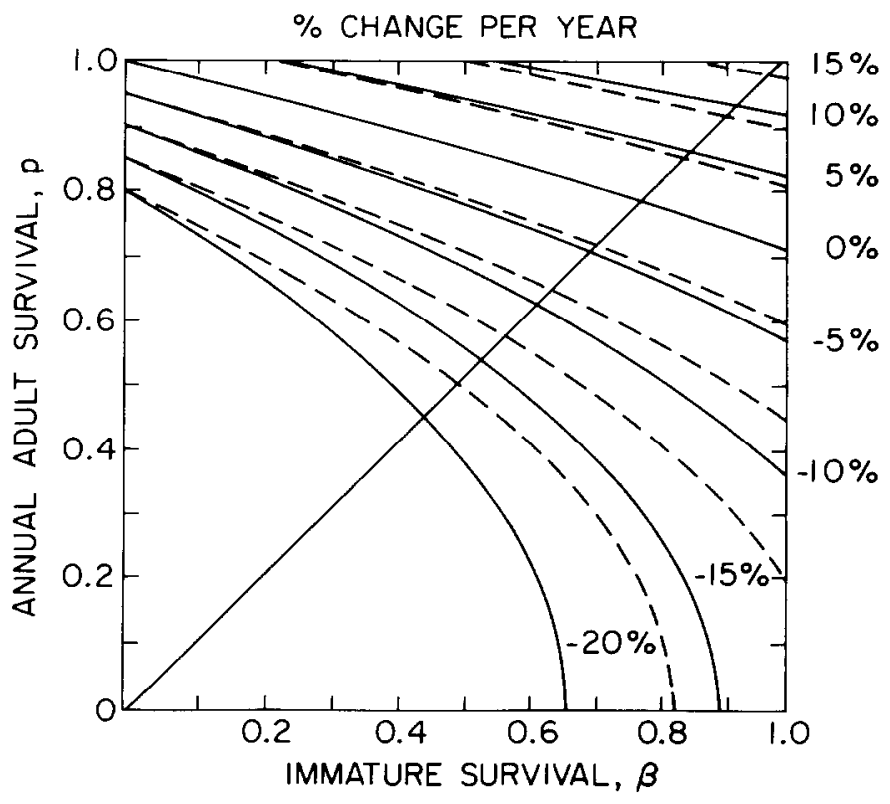

FIG. 1. The effect of quick development on the population growth of the California condor. The solid and dashed lines are constant percentage change isopleths as functions of $\beta$ and $\rho$. The basic life history (with earliest reproduction at age 5) results in the solid isopleths. The dotted lines show the effect of quick development with earliest reproduction at age 4. (a) Mertz's result with $\beta$ compressed from 5 to 4 years; (b) the result when $\beta$ is not compressed. $\beta_{1}$ is the first year mortality for both the basic life history and quick development. (a) is from Mertz [27]. 
(b)

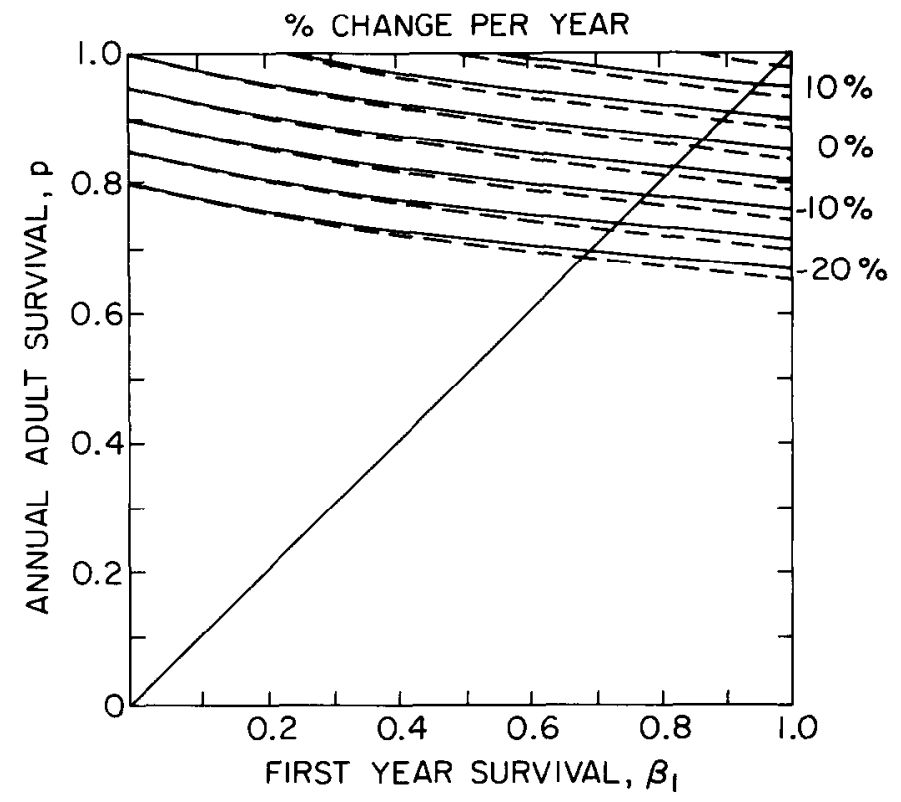

FIG. 1. Continued

of the red-footed booby (Sula sula). If the resulting life table entries are substituted into Equation (1), the result is

$$
\lambda^{\tau}-\rho^{2} \lambda^{\tau-2}-\frac{\beta_{1} \rho^{\tau-1}}{2}=0 .
$$

This equation can then be used to make a new figure corresponding to Mertz's Figure 2 [see Figure 1(b)]. Because the time frame of $\beta$ in Mertz's figure is in question, $\beta_{1}$ is used for the scale on the $x$-axis in Figure $1(b)$. There is no simple scale translation that will allow Figure $1(a)$ and (b) to be superimposed in a meaningful way. In Figure 1(a), when $\tau=5$ (the solid lines), $\beta$ is in units of proportion of young surviving to age 5 . When $\tau=4$ (the dashed lines), $\beta$ is in units of proportion of young surviving to age 4 . In Figure 1(b), $\beta_{1}$ is always in units of proportion of young surviving to age 1 . When the time frame is fixed as in $\beta_{1}$ in Equation (2h) and Figure 1(b), it is seen that earlier development, in the absence of a corresponding acceleration of immature mortality rates, makes the rate of population growth more positive even when the population is declining. Thus, we see that Mertz's claims are correct only when "earlier reproduction" is coupled with earlier immature mortality. Earlier reproduction may sometimes contain an element of earlier immature mortality. 
Nichols et al. [30] used Mertz's approach to examine management implications for the Everglade kite. They reformulated $\beta$ as we did and got results similar to Figure 1(b). In spite of this, they accepted Mertz's results and used them in saying that inferences drawn concerning populations with high fecundity may not apply to populations with low growth rates. They explained the difference in formulation of their prereproductive mortality by saying,

Lewontin [23] and Mertz [27, 28] have considered a development time which is independent of prereproductive mortality, for the purpose of isolating specific effects of these factors.

We find it difficult to envisage that a change in the age of sexual maturity could occur independently of changes in prereproductive mortality, and we have thus combined both effects in our consideration of development-time.

But prereproductive mortality occurs over a specific span of time-the development time. In Mertz's formulation, when development time is changed, prereproductive mortality occurs over the new span of time and the mortality rate is changed. In terms of the life table functions $l_{x}$ and $m_{x}$, the treatment of Nichols et al. is independent and that of Mertz is dependent. In the independent treatment, the question of how a change in development time affects mortality rates is then left to be treated in an explicit fashion.

Goodman [15] explained that what Mertz had done was simply to distort the time scale of the life history so that regardless of whether the population were increasing or declining it would do so faster. However, Mertz's result has also been cited by Wiley [40] as a special case showing that "in decreasing populations deferred reproduction does not necessarily reduce $r$, ...even though other life-history parameters remain unchanged." Of course, other life history parameters have changed. Although Mertz's result is not incorrect if Goodman's [15] explanation is used, it has often been misunderstood. Many published references to Mertz [27] resemble Wiley [40] as quoted but with the word "necessarily" left out $[2,4,5,10$, $13,16,19,20,27,28,34,39,41]$.

\section{EXAMPLE II}

It has been common to avoid mathematical difficulty by lumping $l_{x}$ and $m_{x}$ together into a single variable $v_{x}\left(=l_{x} m_{x}\right)$. There is nothing wrong with this by itself; however, results are then often presented in terms of the timing of reproduction without reporting the fact that mortality rates are also being changed. When reproduction is moved to an earlier age, the mortality rates are increased so that $v_{x}$ has the same value at that earlier age. Lewontin [23] used this formulation and then referred to the changes he made in the $v_{x}$ curve as "reducing development time" and "decreasing the age to sexual maturity." Although in the beginning of the paper he did mention the incorporation of mortality rates, this was not stressed in the later discussion. If this approach were naively applicd to declining populations, it would be 
tempting to reach the erroneous conclusion that "reducing development time" causes the population to decline more rapidly. MacArthur and Wilson [25] used Lewontin's model without reiterating that "changing the age to sexual maturity" involved a quantitative change in fecundity or a shift in mortality rates to maintain a constant $R_{0}$. Green and Painter [17] also used $v_{x}$ and referred to a shift in $v_{x}$ as changing "development time." Costello and Taylor [9] used a similar formulation. They concluded that "increasing the age at first reproduction yields diminishing returns in reduction of $r$ as $r$ comes close to 0 ," and when $r=0$, "the age at first reproduction matters not." This conclusion is correct only when the interdependence of the variables is made explicit. In order for their "family size" $\left(R_{0}\right)$ to remain constant when reproduction is delayed, mortality rates must be decreased or fecundity increased. The net maternity function, $v_{x}$, accomplishes this by lumping survival and fecundity together and then shifting them as a unit. This approach applied to declining populations would give the same conclusions as Mertz [27, 28].

Some authors have maintained $l_{x}$ and $m_{x}$ as separate variables in their analyses $[6,11,21]$. By doing so, they have kept mortality assumptions explicit.

\section{EXAMPLE III}

A similar situation arises in MacArthur and Wilson's discussion [25, p. 88] of the equation

$$
r \cong \frac{\ln R_{0}}{M}
$$

where $M$ is the mean age at reproduction. They made the following statement:

Even this oversimplified formula lets us clear up one common misconception. It confinus that when $R_{0}$ is large, reducing $M$ is the easiest way to increase $r$, as Lewontin's models indicated. However, when $R_{0}$ is near 1 , a reduction in mean age at reproduction will increase $r$ only to an insignificant extent.

Although they did not mention declining populations $\left(R_{0}<1\right)$, their reasoning might suggest the conclusion that in declining populations "a reduction in mean age at reproduction" would cause the population to decline more rapidly. The validity of this claim depends on the relationships between $r, R_{0}, M$, and the life table. Their implicit assumption that $R_{0}$ remains constant requires that the mortality rates be increased as the fecundity is shifted to earlier ages. This is not simply "a reduction in the mean age at reproduction." If only the fecundity were shifted, then $R_{0}$ would increase as $M$ decreases. The overall effect would be that as reproduction is moved to 
earlier ages, $r$ becomes more positive even in cases where $r$ is zero or negative. This conclusion (which is actually correct) seems to be their unstated "common misconception."

\section{EXAMPLE IV}

Stenseth [37] and Pianka and Parker [32] also exemplify the interdependence of variables in their analysis of an organism's choice between current and future reproduction. Their analysis begins with Fisher's [12, p. 27] equation for the reproductive value of an individual at age $x$ :

$$
v_{x}=\frac{1}{l_{x}} \sum_{t=x}^{w} e^{-r(t \cdot x)} l_{t} m_{t},
$$

where $w$ is the age at last reproduction. The reproductive value is then partitioned into two parts:

$$
v_{x}=m_{x}+e^{-r} v_{x}^{*},
$$

where $m_{x}$ represents present progeny and $v_{x}^{*}$ is the "residual reproductive value" defined by

$$
v_{x}^{*}=\frac{l_{x+1}}{l_{x}} v_{x+1} .
$$

Equation (5) is rearranged to give

$$
v_{x}^{*}=e^{r} v_{x}-e^{r} m_{x},
$$

which is then used in a graphical analysis to determine the optimal allocation of reproductive effort between current and future progeny as shown in Figure 2. Using empirical or hypothetical data, the relationship between $m_{x}$ and $v_{x}^{*}$ is determined for an individual at a particular age. This relationship is given by the solid line. Any solution representing a choice of $m_{x}$ and a resulting $v_{x}^{*}$ must fall on this line. The object is to choose the value of $m_{x}$ that results in the maximum value for $v_{x}^{*}$. Equation (7) gives a relationship for $m_{x}, v_{x}$, and $v_{x}^{*}$, but it requires a value for $r$. Stenseth [37] determines the value of $r$ for a given population and enters it into Equation (7), fixing the slope of the dashed line in Figure 2. The dashed line is then moved perpendicularly (so that the slope is unchanged) until it just touches the solid line. The point of contact is the solution for $m_{x}$ that maximizes $v_{x}$, because the dashed line going through that point has the largest intercept value of any line with the same slope that touches the solid curve. If the solid curve were represented by an equation, an alternative method for finding the maximum $v_{x}$ would be to use partial derivatives. 


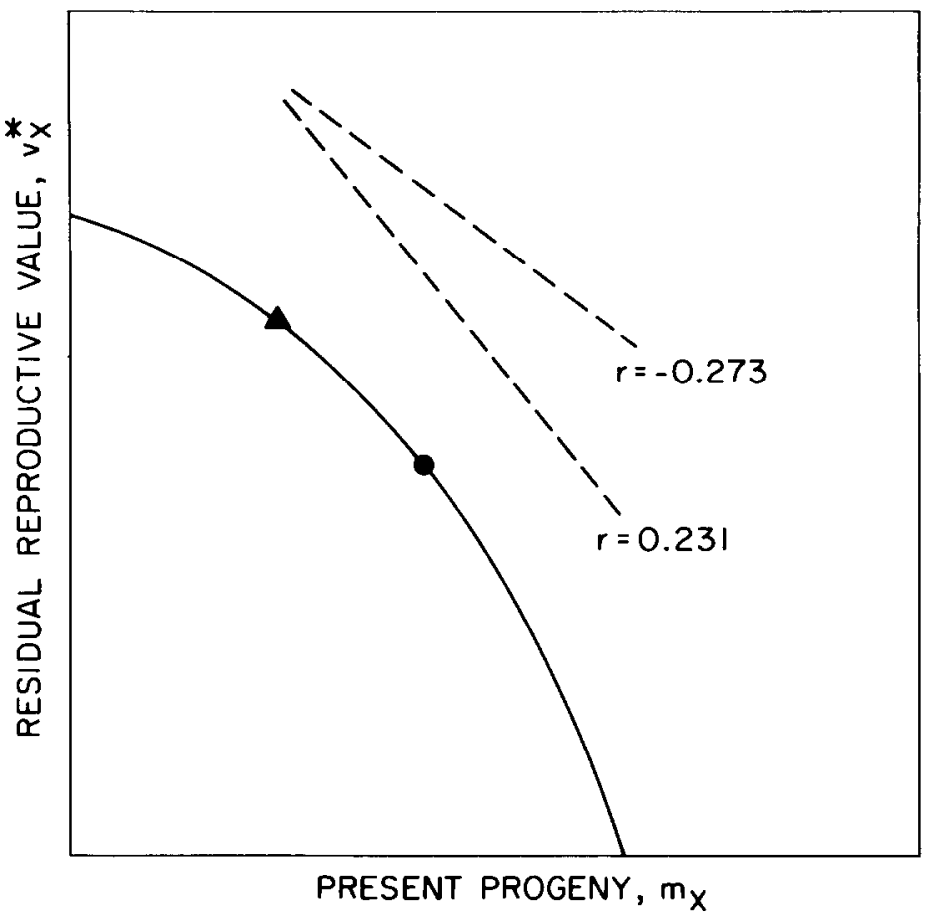

FIG. 2. Expectation of future reproductive success, or residual reproductive value, is plotted against current reproductive output for a hypothetical case. The solid line represents the assumption that as an individual's current reproductive effort increases, the expenditure of resources reduces its expectation of future reproductive success. The dashed lines represent families of parallel lines from Equation (7), where a value of $r$ determines the slope of the family of lines and a value of $v_{x}$ determines the intercept for particular line. The dot represents the combination of $m_{x}$ and $v_{x}^{*}$ which gives the maximum value for $v_{x}$ as determined by the family of lines for $r=0.231$. The triangle is a similar combination for $r=-0.273$. Modified from Stenseth [37].

The graphic analysis in Figure 2 can be done for any given value of $r$ and led Stenseth to the conclusion that "allocating relatively more resources to current reproduction is the optimal strategy in a growing population." The same analysis would lead to the conclusion that in a declining population reproductive effort should be saved for future progeny. This is similar to the examples described previously. To reach Stenseth's conclusion, changes in age dependent survivorship and fecundity must be considered. The values for $r$ are based on and derived from an assumed constant life history. When a change is made in allocation to current versus future offspring, the life history changes and $r$ changes. Indeed, if $r$ did not change, there could be no 
long term selective advantage to changing one's life history (assuming of course that such a change is passed on to one's progeny).

Pianka and Parker [32] presented a slightly different version of the same model. They chose to discuss only populations in which $r=0$. Since $r=0$, $e^{-r}=1$ and drops out of all their equations. In fact, they never give a version of the equations with $r$ included and only mention in passing that for a population changing in size (i.e. $r \neq 0$ ) the equation is slightly more complicated. Because $r$ was eliminated only in appearance by the requirement that it be 0 , the formulation of their model is the same as Stenseth's and is subject to the same limitations.

\section{EXAMPLE V}

Bell [1] analyzed the effect of age at maturity on the rate of increase of a population. The simplified case that he discussed at the bottom of p. 69 is for a population with the life history shown in Table 2 . The values from the life table are substituted into Equation (1) to arrive at

$$
1=e^{-r a} s c^{a-1} b\left[1+\frac{c}{e^{r}}+\left(\frac{c}{e^{r}}\right)^{2}+\cdots\right],
$$

which is simplified to

$$
1=e^{-r a} s c^{a-1} b\left[1-\frac{c}{e^{r}}\right]^{-1}
$$

and rearranged to

$$
e^{r a}-c e^{r(a-1)}-s c^{a-1} b=0
$$

\section{TABLE 2}

A Simple Iteroparous Life Table ${ }^{a}$

\begin{tabular}{lll}
\hline$x$ & $l_{x}$ & $m_{x}$ \\
\hline 0 & 1 & 0 \\
1 & $s$ & 0 \\
2 & $s c$ & 0 \\
$\vdots$ & $\vdots$ & $\vdots$ \\
$a-1$ & $s c^{a-2}$ & 0 \\
$a$ & $s c^{a-1}$ & $b$ \\
$a+1$ & $s c^{a}$ & $b$ \\
$\vdots$ & $\vdots$ & $\vdots$ \\
$x>a$ & $s c^{x-1}$ & $h$ \\
\hline
\end{tabular}

a Made up from the example used by Bell [1, p. 69]. 
In Equation (8) Bell had the summation terminate at age $w$, but the simplification to Equation (9) assumes $w \rightarrow \infty$. This is an important point, and a later section of this paper will deal with finite versus infinite summations. Implicit differentiation of Equation (10) yields

$$
\frac{d e^{r}}{d a}=\frac{s b c^{a-1} \ln c}{e^{r(a-1)}\left[a-c(a-1) e^{-r}\right]} .
$$

The numerator of this equation must be negative, since $c<1$. If the derivative is to be positive, that is, if an increase in the age at maturation is to have a positive effect on the rate of increase of the population, then the denominator must also be negative. For that to be the case $c(a-1) e^{-r}$ must be greater than $a$. Rearranging, $e^{r}(=\lambda)$ must be less than $c(a-1) / a$. From this, Bell concludes that " a heritable tendency for delayed maturity may be selected... if the population is decreasing in numbers rather rapidly." What must be noted is that for this example, even if there is no reproduction, the population cannot decline faster than the death rate. After the first year, if there is no reproduction, $s$ is no longer important and the population will decline at a rate $e^{r}=\lambda=c$. This can be seen intuitively or it can be derived from Equation (10) by setting $b=0$. The condition for the derivative $d e^{r} / d a$ to be positive is thus impossible in the context of this model. In the context of this model a correct conclusion is that a decrease in the age at first reproduction will always cause a positive change in $\lambda$.

Demetrius [11, p. 134] derived a similar but more detailed inequality using the Leslie matrix formulation. His inequality is

$$
\frac{\partial \lambda}{\partial F_{i}}>\frac{\partial \lambda}{\partial F_{j}}, \quad i<j, \quad \text { for } \quad \lambda>P=\max \left(P_{i}\right),
$$

where $F_{i}$ is the fecundity at age $i$ and $P_{i}$ is the probability that an individual age $i$ will survive to age $i+1$. This shows that a given increase in fecundity will have a greater positive effect on the growth rate if it occurs at an earlier age. The result applies to increasing or stable populations and to declining populations in which $\lambda>P$. It leaves other cases where $\lambda<P$ unresolved. Caswell [6] gives a slightly stronger proof that applies if and only if $\lambda>P_{i}$. Both Demetrius and Caswell were working with a formulation that allows the $P_{i}$ to vary with age and that does not assume an infinite life span. This is different from Bell's model, and under these circumstances $\lambda$ can be less than a particular $P_{i}$. Infinite life span will be discussed in more detail below.

\section{EXAMPLE VI}

Mertz [28] examines the consequences of various changes in life history. Among the equations used are Equation (1) in this paper, an extended 
version of Equation (3) in this paper, and $g_{x}=e{ }^{x} l_{x} m_{x}$, the "probability density function for the age of reproducing females in an infinite stable population." According to Mertz [28], these three equations

show that a lengthening of development lowers $r$ for growing populations. produces no effect for $r=0$, and actually increases $r$ (makes it less strongly negative) for decreasing populations. In other words, a lengthening of development, which is ordinarily opposed by selection, may be favored in declining populations inasmuch as it slows the rate of population decline.

Although the reason for these conclusions is not stated, they seem to be based on the division by $M$ in Equation (3) and on the appearance of $e^{-r x}$ in the other two equations. The interpretation of Equation (3) has already been discussed in Example III above. The interpretation of $e^{-r x}$ was mentioned in the introduction and should be clear from other discussions throughout this paper.

The reader of Mertz [28] could easily believe that the spacing of births will be dealt with independently from death rates. However, Mertz concludes that a change in prereproductive period in itself has no effect on $R_{0}$. For this is to be true, there must be a corresponding change in mortality or the magnitude of the fecundity must change. The quotation above is valid only in very limited conditions of complete interdependence of fecundity and mortality that should be made explicit in each case. Nonetheless, it has been used to explain or support results in both theoretical and applied studies by a number of other authors $[2,4,5,7,8,10,13,14,19,20,29,41]$. It has also appeared in reviews by Stearns $[35,36]$, and similar statements have been made independently by Laughlin [22] and Hamilton [18].

The factor $e^{-r x}$ appears in many of the standard demographic equations. The analogy between it and discount rates has caused repeated difficulties in interpretation. These difficulties have occurred most of ten in dealing with reproductive values and are exemplified by Laughlin [22]:

... in a decreasing population the older mothers actually make the most important contribution to reproduction.

\section{Hamilton [18]:}

For an organism which reproduces repeatedly the concept of fitness is not so casily defined. The expectation of offspring suffers from the objection that early births are worth more than late in an increasing population, and vice versa in a decreasing one....

and Hirshfield and Tinkle [19]:

In a declining population. of fspring produced late in a female's life would contribute more to her fitness

(cited Mertz [28] and Hamilton [18]). These three quotations are all in reference to reproductive value as a measure of fitness (cf. [12, p. 27]) However, the reproductive value is derived assuming constant life history 
functions. It gives the relative contribution an individual currently of age $x$ will make to future generations. It does not refer to the contribution of particular offspring to the fitness of that individual. Reproductive values are useful in discussions of colonizing propagules, exploitation, and perhaps the effects of selective pressures at different ages (as suggested by Fisher). It is tempting to conclude, using $r$ as a discount rate, that later offspring contribute more to an individual's fitness in a declining population. But this is incorrect, because if we change the life history by adding later offspring or earlier offspring, $r$ will change. If the change in life history is a heritable attribute, then the question of fitness is whether this individual's descendants, given their own fixed life history, will increase in numbers more rapidly than will the remainder of the population, which is determined by the difference in $r$. A similar argument also applies to Stenseth's [37] analysis, which is discussed in Example IV above.

To determine the age at which added reproductive effort will yield the greatest selective advantage, we should be looking at the change in growth rate resulting from a given change in reproduction at different ages. In other words, we should be looking at $\partial \lambda / \partial m_{x}$ as a function of $x$. The age $x$ at which this partial derivative has the largest positive value is the age at which reproductive effort should be added. Analyses of this partial derivative have been published by Demetrius [11] and Caswell [6] and were discussed at the end of Example $\mathrm{V}$ above.

\section{DISCUSSION}

\section{A. EVOLUTIONARY ASPECTS OF THE RATE OF CHANGE OF A POPULATION}

Mertz's [28] paper centers around the evolution of life history strategies in Tribolium. In laboratory culture conditions they go through repeated cycles of rapid growth in fresh culture medium followed by prolonged periods of stable or declining populations. Dawson [10] pointed out that similar population dynamics may occur in the natural habitat of Tribolium. Mertz argued that the life history of Tribolium has been shaped by selection during these repeated long periods of decline. He claimed that during a decline, increased development time slows the rate of decline and is therefore selectively advantageous.

Since the above argument is incorrect, other biological explanations must be found. Mertz [28] pointed out the high incidence of cannibalism on eggs and larvae in dense populations. It seems clear that the reproductive behavior of Tribolium is a selective response to short periods of abundant resources followed by longer periods of crowding and intense competition for resources. Pianka [31] argued that "in order to maximize overall lifelong contribution to future generations, an optimal organism should weigh its 
immediate prospects of reproductive success against its long-term future prospects." (Pianka cited Williams [38] and Pianka and Parker [32].) In this light, when the population is crowded and the rate of cannibalism on eggs and larvae is high, it is advantageous to an individual beetle to delay reproduction and put its resources into its own survival. Then, at some future point when the competition has died down or the individual has emigrated, it can reproduce with a higher likelihood of success. The declining population does not lead to delayed reproduction; rather the reverse is true. Cannibalism and delayed reproduction in response to overcrowding cause a population decline.

The above argument can be applied to $r$ and $K$ selection in general. An organism's evolutionary objective is to obtain resources and convert them into offspring. If there is an abundance of resources available, emphasis can be put into reproduction and the result will be an increasing population. If there is heavy competition for resources, then emphasis must be put on obtaining resources and it is likely that a stable or declining population will result. Thus, the rate of change of a population is not a cause of reproductive strategies but rather an effect of reproductive strategies that arise from conditions of resource availability or competition.

\section{B. FINITE VERSUS INFINITE SUMMATIONS}

The usual demographic analysis is based on the assumption of constant life history functions and the consequent stable age distribution that arises under the operation of those functions. For rapidly increasing populations, the stable age distribution is skewed so that the younger individuals form the largest proportion of the population [see Figure 3(a)]. As individuals become older, the age groups to which they belong become less important. Under these circumstances the distinction between finite and infinite series in the mathematical summations (e.g. $w=50$ versus $\infty$ in Bell [1]) is of little importance. The truncated end of the series [on the right in Figure 3(a)] will form a minute fraction of the population being considered. However, for rapidly declining populations, the older age groups can form the most important part of the population [see Figure 3(b)]. The stable age distribution is given by

$$
c_{x}=\frac{e^{-r x} l_{x}}{\sum_{t=0}^{\infty} e^{-r t} l_{t}} .
$$

For the simplest case where $l_{x}=p^{x}$, the numerator of Equation (13) can be rearranged to show that

$$
c_{x}-\left(\frac{P}{\lambda}\right)^{x}
$$

If $P / \lambda$ is greater than 1 , i.e. if $\lambda<P$, then $c_{x}$ will increase as $x$ increases. 

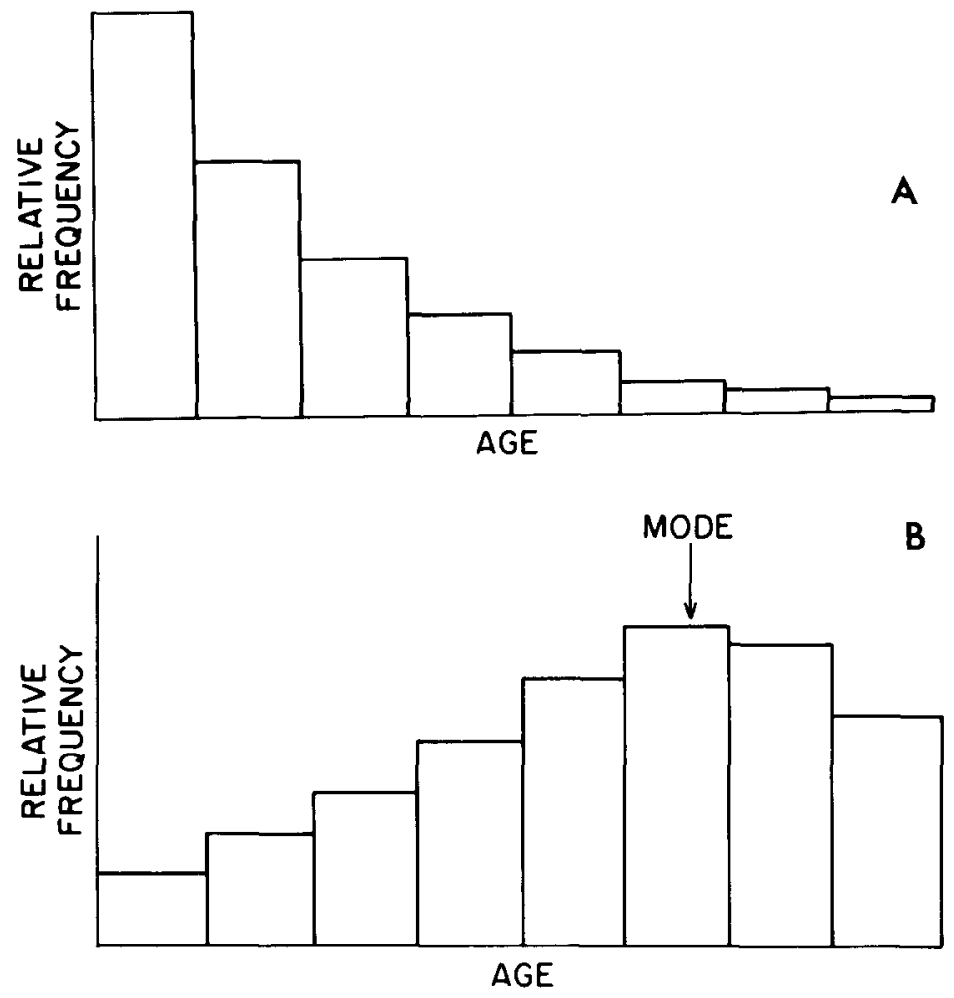

FIG. 3. (a) General form of the stable age distribution for an increasing population. (b) A truncated age distribution (not stable) for a rapidly declining population. In a stable age distribution, the mode would have moved to the right and been truncated. The result would be a mirror image of the distribution for the increasing population.

We noted above (in reference to [1]) that a population with infinite age (no senescence) and a constant death rate $\left(l_{x}=P^{x}\right.$ for $x=0$ to $\left.\infty\right)$ cannot decline at a rate faster than its death rate (i.e. $\lambda$ cannot be less than $P$ ). However, if the population has a finite maximum age, $w$, at which $l_{x}=0$, then the geometric rate of increase, $\lambda$, can be less than $P$. Under such conditions, the older age groups make up a larger portion of the population [see Figure 3(b)], and the oldest age group is dying off entirely at each time interval. Thus, when $\lambda$ is small, the choice between finite and infinite series is no longer simply a question of mathematical nicety. The tails (older age groups) of the summations are no longer negligible portions of the overall population. The choice must therefore be made with careful attention to the biology of the organism that is being studied. 
A related point is that as $\lambda$ approaches $P$, the likelihood that the population will approach a stable age distribution in a reasonable length of time becomes much smaller. When the series of age groups is very long or infinite, the mode of the age distribution would move continuously to older age groups as the population declines [see Figure 3(b)]. A declining population of Tribolium, for example, is unlikely to approach the stable age distribution determined by the modified life history functions that exist during the population decline.

\section{CASES IN WHICH SELECTION MAY FAVOR DELAYED REPRODUCTION}

In some of the cases referred to in this paper, particularly those dealing with infinite series and age independent death rates (no senescence), it is clear that earlier reproduction will always cause the rate of increase to become more positive. However, in dealing with finite series and with age dependent death rates, it is a different matter. Clearly, there is not a symmetry about $r=0$ as claimed by many of the authors cited in this paper. Demetrius [11, p. 134] and Caswell [6] proved that the point of change is where $\lambda=P_{\text {i }}$ (see Example $\mathrm{V}$ above). For example, in the simple life table shown in Table 3, the following cases are instructive:

(a) Let $P=0.9$ and $m=1$. Then $\lambda=0.9$. If $m$ is now moved to age group 3, $\lambda$ is still 0.9 . This is the turnover point at which $\lambda=P$ and the timing of reproduction does not affect $\lambda$.

(b) Now, let $P=0.9$ and $m=0.8$. In this case, $\lambda=0.851$ and is less than $P$. If $m$ is moved to age group $3, \lambda$ changes to 0.835 . This result indicates that when $\lambda$ is less than $P$, earlier reproduction will accelerate the rate of decline.

TABLE 3

A Simple Life Table

\begin{tabular}{lll}
\hline$x$ & $l_{x}$ & $m_{x}$ \\
\hline 0 & 1 & 0 \\
1 & $P$ & 0 \\
2 & $P^{2}$ & 0 \\
3 & $P^{3}$ & 0 \\
4 & $P^{4}$ & $m$ \\
5 & 0 & 0 \\
\hline
\end{tabular}


The biological importance of cases such as the one just described has yct to be established. If the life histories are fixed, then populations that are declining at rates such that $\lambda<P$ for a substantial proportion of time are in exceedingly precarious positions with respect to selection and extinction. Even granting that the condor may have been declining for a thousand years, it cannot have been declining fast enough for this condition to apply. If a population of 10,000 condors were declining at a rate of $\lambda=0.95$, there would only be 59 of them in 100 years and they would be extinct in 200 years. If $\lambda$ were 0.90 , they would be extinct in 100 years.

Not only are these cases precarious with respect to extinction, but they would appear to be evolutionarily unstable. For the cases to which this applies, $\lambda=P$ is a turnover point. If $\lambda<P$, selection favors delayed reproduction. If $\lambda>P$, selection favors earlier reproduction. If $\lambda<P$, an individual that makes a heritable gain in reproduction might achieve a growth rate for its progeny that would reverse the direction of selection. This instability would be accented by variability in the population.

If life histories are variable or there is behavioral plasticity, as has been suggested for Tribolium [28], then there are demographic difficulties with the stable age distribution as have been pointed out above. In addition, Tribolium population cycles have been described as rapid increases followed by longer periods of slow decline [28]. Granting that "slow" and "rapid" are subjective, a "very" rapid decline in which $\lambda<P$ is required for selection to favor delayed reproduction from a purely demographic point of view.

It should also be pointed out that the rate of selection may be different during the periods of increase and decline. Therefore, the length of those periods alone is not sufficient to tell which, if either, will dominate in the evolutionary history of an organism.

\section{SCALING EFFECTS IN INCREASING POPULATIONS}

This paper clarifies a number of related concepts in the demography of declining populations. However, by altering the scale of effects, these concepts can be applied to increasing and stable populations as well. For example, Costello and Taylor [9] examined population strategies for humans and the question of reducing $r$ by increasing the age at first reproduction. They claimed that as $r$ approached 0 the effect of further increases in the age at first reproduction would be less, and when $r$ equaled 0 the age at first reproduction would not matter. They obtained this result by lumping survival and fecundity together so that an "increase in the age at first reproduction" implicitly involved increased fertility and/or increased survival in order to maintain the same family size $\left(R_{0}\right)$. In the absence of these implicit changes, increasing the age at first reproduction should achieve greater gains in reduction of $r$ than they suggested and there should still be gains in reduction of $r$ when $r=0$. Busby and Mode [3] examined the effects of age 
of marriage for human populations and showed a numerical example in which a declining population declined more rapidly when reproduction was delayed. This is particularly important and has implications for government policies since human populations have the flexibility of choice and can affect age at first reproduction without altering the biology of development up to reproductive age.

William D. Hamilton and Richard D. Alexander provided valuable advice and support. In addition, the following people read various drafts of this paper and supplied useful comments and suggestions: Bobbi S. Low, Alvin L. Jensen, Gary E. Belovsky, Steven R. Beissinger, Margaret R. Halbeisen, Barry Chernoff, Godfrey R. Bourne, H. Lisle Gibbs, Stephen Dodge, and C. J. Mode.

\section{REFERENCES}

1 G. Bell, On breeding more than once, Amer. Natur. 110:57-77 (1976).

2 J. F. Boyer, Reproductive compensation in Tribolium casteneum. Evolution 32:519 (1978).

3 R. C. Busby and C. J. Mode. Theory and applications of a computationally efficient cohort simulation model of human reproduction. Math. Biosci. 64:45-74 (1983).

4 T. V. Callaghan, Growth and population dynamics of Carex bigelowii in an alpine environment, Oikos 27:402-413 (1976).

5 T. V. Callaghan and N. J. Collins, Strategies of growth and population dynamics of tundra plants, Oikos 27:383-388 (1976).

$6 \mathrm{H}$. Caswell, A general formula for the sensitivity of population growth rate to changes in life history parameters, Theoret. Population Biol. 14:215-230 (1978).

$\%$ H. Caswell and A. Hastings. Fecundity, development time, and population growth rate: An analytical solution. Theoret. Population Biol. 17:71-79 (1980).

8 H. Caswell and P. A. Werner, Transient behavior and life history analysis of teasel (Dipsacus shloestris Huds.). Ecologr 59:53-66 (1978).

9 W. G. Costello and H. M. Taylor, Deterministic population growth models, Amer. Math. Monthly 78:841-855 (1971).

10 P. S. Dawson, Life history strategy and evolutionary history of Tribolium flour beetles. Evolution 31:226-229 (1977).

11 L. Demctrius, The sensitivity of population growth rate to perturbations in the life cycle components, Math. Biosci. 4:129-136 (1969).

12 R. A. Fisher, The Genetical Theory of Natural Selection, Oxford U.P., 1930.

13 N. R. French, B. G. Maza, H. O. Hill, A. P. Aschwanden, and H. W. Kaaz, A population study of irradiated desert rodents. Ecol. Monogr. 44:45-72 (1974).

14 J. T. Giesel, Fitness and polymorphisms for net fecundity distribution in iteroparous populations, Amer. Natur. 108:321-331 (1974).

15 D. Goodman, Natural selection and a cost ceiling on reproductive effort, Amer. Natur. $108: 247-268(1974)$.

16 R. F. Green, $\Lambda$ note on $K$-selection, Amer. Natur. 116:291 296 (1980).

17 R. Green and P. R. Painter, Selection for fertility and development time, Amer. Natur. 109:1-10)(1975) 
18 W. D. Hamilton. The moulding of sencsecnce by natural selection, J. Theoret. Biol. $12: 12-45$ (1966).

19 M. F. Hirschfield and D. W. Tinkle, Natural selection and the evolution of reproductive effort, Proc. Nat. Acad. Sci. U.S. A. 72:2227-2231 (1975).

20 R. D. Howard, Estimating reproductive success in natural populations, Amer. Natur. 114:221-231 (1979).

21 N. Keyfitz, Linkages of intrinsic to age-specific rates, J. Amer. Statist. Assoc. 66:275-281 (1971).

22 R. Laughlin, Capacity for increase: A useful population statistic, J. Anim. Ecol. 34:77-91 (1965).

23 R. C. Lewontin, Selection for colonizing ability, in The Genetics of Colonizing Species (H. G. Baker and G. L. Stebbins, Eds.), Academic, New York, 1965, pp. 77-94.

24 A. J. Lotka, Elements of Physical Biology, Williams and Wilkins, 1925.

25 R. H. MacArthur and E. O. Wilson, The Theory of Island Biogeography, Princeton U.P.. 1967.

26 D. B. Mertz, Notes on methods used in life-history studies, in Readings in Ecology and Ecological Genetics (J. H. Connell, D. B. Mertz, and W. W. Murdoch, Eds.), Harper and Row, New York, 1970, pp. 4-17.

27 D. B. Mertz, The mathematical demography of the California condor population, Amer. Natur. 105:437-453 (1971).

28 D. B. Mertz, Life history phenomena in increasing and decreasing populations, in Statistical Ecology, Vol. 2 (G. P. Patil, E. C. Pielou and W. E. Waters, Eds.), Pennsylvania State U.P., 1971, pp. 361-399.

29 D. B. Mertz, Senescent decline in flour beetle strains selected for early adult fitness, Physiol. Zool. 48:1-23 (1975).

30 J. D. Nichols, G. L. Hensler, and P. W. Sykes, Jr., Demography of the Everglade kite: Implications for population management, Ecol. Model. 9:215-232 (1980).

31 E. R. Pianka, Natural selection of optimal reproductive tactics, Amer. Zool. 16:775-784 (1976).

32 E. R. Pianka and W. S. Parker, Age-specific reproductive tactics, Amer. Natur. 109:453-464 (1975).

33 R. E. Ricklcfs, Ecology, 2nd cd., Chiron, 1979.

34 J. J. Sohn, Socially induced inhibition of genetically determined maturation in the platyfish, Xiphophorus maculatus, Science 195:199-201 (1977).

35 S. C. Stearns, Life-history tactics: A review of the ideas, Quart. Rev. Biol. 51:3-47 (1976).

36 S. C. Stearns, The evolution of life history traits: A critique of the theory and a review of the data, Annual Rev. Ecol. Syst. 8:145-171 (1977).

37 N. C. Stenseth, Demographic strategies in fluctuating populations of small rodents, Oecologia 33:149-172 (1978)

38 G. C. Williams, Adaptation and Natural Selection, Princeton U.P., 1966.

39 D. R. Strong, Jr. and M. D. Wang, Evolution of insect life histories and host plant chemistry: Hispine beetles on Heliconia, Evolution 31:854-862 (1977).

40 R. H. Wiley, Effects of delayed reproduction on survival, fecundity, and the rate of population increase, Amer. Natur. 108:705-709 (1974).

41 J. R. Ziegler, Evolution of the migration response: Emigration by Tribolium and the influence of age, Evolution 30:579-592 (1976). 